\title{
A Educação Permanente como ferramenta estratégica na Coordenação em Saúde Mental
}

\author{
Permanent education as a strategic tool in Coordination in Mental Health \\ La educación permanente como herramienta estratégica em la Coordinación em Salud Mental
}

Déborah Leonardo de Souza Martins ${ }^{1 *}$, Ana Lúcia Abrahão²

Como citar esse artigo. Martins, DLS; Abrahão, AL. A Educação Permanente como ferramenta estratégica na Coordenação em Saúde Mental. Revista Pró-UniverSUS. 2019 Jul./Dez.; 10 (2): 128-130.

\section{Resumo}

Objetivo: Reconhecer os elementos empregados pelos trabalhadores de saúde mental na construção do cuidado, junto aos usuários de um Centro de Atenção Psicossocial Infantil. Metodologia: trata-se de uma pesquisa de abordagem qualitativa que utilizará como referencial teórico-metodológico a Sócioclínica Institucional em sua modalidade de intervenção a análise institucional das práticas profissionais. A pesquisa intervenção será realizada no Centro de Atenção Psicossocial Infanto-Juvenil de um município do Estado do Rio de Janeiro, tem como critério de inclusão a participação dos trabalhadores que atuam há mais de um ano no serviço de saúde mental, com vínculo estatutário e critério de exclusão, os trabalhadores que se encontrarem de licença e férias. Os dados serão coletados através da realização de encontros com os trabalhadores e entrevistas narrativas. O tratamento dos dados coletados será realizado por meio da identificação de eixos de análises centrados na socioclinica institucional. Resultados esperados: implantar a Educação Permanente em Saúde como ferramenta estratégica para reflexão crítica do processo de trabalho e desenvolvimento dos trabalhadores de saúde mental do município estudado

Palavras-chave: Profissionais de Saúde, Saúde Mental, Fluxo de Trabalho, Educação Permanente.Mental.

\begin{abstract}
Objective: To recognize the elements employed by mental health workers in the construction of care, together with the users of a Child Psychosocial Care Center. Methodology: This is a qualitative approach research that will use as a theoreticalmethodological framework the Institutional Socioclinic in its modality of intervention the institutional analysis of professional practices. The intervention research will be conducted at the Center for Psychosocial Care for Children and Youth of a city in the state of Rio de Janeiro, has as inclusion criteria the participation of workers who have been working in the mental health service for over a year, with statutory bond and criteria exclusion, workers on leave and vacation. Data will be collected through meetings with workers and narrative interviews. The treatment of the collected data will be accomplished through the identification of analysis axes centered in the institutional socioclinica. Expected results: to implement Permanent Health Education as a strategic tool for critical reflection of the work process and development of mental health workers of the studied municipality.
\end{abstract}

Keywords: Health Personnel, Mental Health, Workflow, Permanent Education.

\section{Resumen}

Objetivo: reconocer los elementos empleados por los trabajadores de salud mental en la construcción de la atención, junto con los usuarios de un centro de atención psicosocial infantil. Metodología: se trata de un enfoque de investigación cualitativa que utilizará como marco teórico-metodológico el Socioclínico Institucional en su modalidad de intervención el análisis institucional de las prácticas profesionales. La investigación de intervención que se llevará a cabo en el Centro de Atención Psicosocial para Niños y Jóvenes de una ciudad en el estado de Río de Janeiro, tiene como criterio de inclusión la participación de trabajadores que han estado trabajando en el servicio de salud mental durante más de un año, con un vínculo y criterio legal. Exclusión, trabajadores con licencia y vacaciones. Los datos se recopilarán mediante reuniones con trabajadores y entrevistas narrativas. El tratamiento de los datos recopilados se logrará mediante la identificación de ejes de análisis centrados en la socioclínica institucional. Resultados esperados: implementar la Educación Permanente en Salud como una herramienta estratégica para la reflexión crítica del proceso de trabajo y el desarrollo de los trabajadores de salud mental del municipio estudiado.

Palabras clave: Trabajadores de salud, Salud Mental, Flujo de Trabajo, Educación Permanente.

Afiliação dos autores: 1. Psicóloga da Prefeitura Municipal de Barra do Piraí. Mestranda do Mestrado Profissional em Ensino na Saúde/ MPES. Universidade Federal FluminenseUFF/Niterói; Brasil. Email:deborahmartins32@yahoo.com ORCID: http://orcid.org/0000-0001-7730-1504

2. Enfermeira. Pós Doutora em Enfermagem. Professora Titular, Programa de Mestrado Profissional em Ensino na Saúde/MPES, UFF, RJ, Brasil. Email:abrahaoana@gmail.com ORCID: https://orcid.org/0000-0002-0820-4329

\footnotetext{
* Email de correspondencia: deborahmartins32@yahoo.com
} 


\section{Introdução}

No Brasil, conquistas como a aprovação da Lei $10.216^{1}$ de 2001, que dispõe sobre a proteção e os direitos de pessoas portadoras de transtornos mentais e redireciona o modelo de assistência, provocou efetivas mudanças no cenário do cuidado em saúde mental. De acordo com a Portaria $336^{2}$ de 2002, foram estabelecidos os Centros de Atenção Psicossocial (CAPS), em várias modalidades definidos segundo porte e complexidade dos territórios. A Rede de Atenção Psicossocial (RAPS) foi instituídacom o objetivo de integrar e criar ações de saúde mental no âmbito do Sistema Único de Saúde (SUS) (Portaria 3.088/2011)

A RAPS, incorpora ao campo da saúde mental, mudanças nas práticas e saberes que perpassa por uma construção histórica que determina o modo de operar dos trabalhadores. Segundo Amarante ${ }^{4}$, o processo da Reforma Psiquiátrica é de alta complexidade social, uma vez que no Brasil a assistência em psiquiatria foi marcada pela violação dos direitos humanos, utilização de tratamentos violentos e arcaicos e segregação da loucura. As discussões sobre o modelo hospitalocêntrico ganharam forças a partir das denúncias de violência trazidas por trabalhadores e grupos de familiares dos internos ${ }^{4}$.

A realização desta pesquisa se faz pertinente por se tratar de uma análise dos fatores implícitos no processo de trabalho em saúde mental, sendo estes determinantes para a concepção das práticas que vem sendo desenvolvidas no campo. No contexto das políticas de saúde mental, as pesquisas já realizadas focam a formação do profissional e a necessidade de adequação dos currículos das universidades às reais necessidades deste trabalho. Contudo é necessário compreender sobre as práticas de saúde que ocorrem num processo de subjetivação do trabalhador, nas relações que estabelece com o usuário, instituição, articulação com a rede e com as ferramentas usadas para exercer o cuidado.

No cotidiano do trabalho as práticas em saúde são reconhecidas como atos produtivos, pois modificam alguma coisa e produz algo novo nos encontros entre agente consumidor (usuário) que traz consigo sua intencionalidade - objeto do ato produtivo e o agente produtor (trabalhador), com suas ferramentas e concepções ${ }^{3}$.

A Educação Permanente em Saúde tem como objetivo provocar uma reflexão sobre o trabalho como ato formativo, a reconhecer o SUS como produto vivo e ativo do cuidado/descuidado nos encontros que vão acontecendo no cotidiano do trabalho. Desta forma oportunizando o aprendizado, que vem sendo construído, naquilo que é produzido junto com o outro e que exige pensar sobre o meu próprio ato 5 .

Objetivos: reconhecer os elementos empregados pelos trabalhadores de saúde mental na construção do cuidado, junto aos usuários de um Centro de Atenção Psicossocial Infantil Propondo-se a realizar um registro das ferramentas utilizadas pelos profissionais que atuam na Rede de Atenção Psicossocial do município; a descrever quais fatores tem sido determinantes para as práticas de saúde que vem exercendo e construir estratégias para que a Educação Permanente possa ser uma ferramenta de fortalecimento desses trabalhadores.

\section{Materiais e métodos}

Trata-se de uma pesquisa de abordagem qualitativa que utilizará como referencial teórico-metodológico a Sócioclínica Institucional, em sua modalidade de intervenção a análise das práticas profissionais.

A pesquisa intervenção se desenvolverá de acordo com a encomenda, sendo que esta pode se modificar a medida que for analisada, de acordo com as demandas que surgirem nos encontros a serem realizados com os trabalhadores. Essa perspectiva exige que não ocorra apenas a análise dos fatos, mas que seja levado em consideração a análise das implicações (vínculos e relações mantidas com e nas instituições), e por fim a elucidação dos analisadores ${ }^{6}$. A proposta é realizar encontros, como rodas de conversa, que oportunizem a análise da narrativa e que possibilite vir a tona as vivencias dos participantes da pesquisa, bem como acerca dos fatores situacionais ${ }^{5}$. A ideia é que as falas a respeito do cotidiano do trabalho sejam claras e carregadas de experiência do participante da pesquisa, permitindo que através da análise das mesmas seja possível a pesquisadora refletir sobre as diferentes perspectivas do trabalhador e de seu modo de cuidar.

A pesquisa será realizada no CAPSI, dispositivos da Rede da Atenção Psicossocial do município do Estado do Rio de Janeiro. Serão incluídos na pesquisa os trabalhadores que atuam há mais de um ano no serviço de saúde mental e como critério de exclusão, os trabalhadores que se encontrarem de licença ou férias. Por se tratar de uma pesquisa que envolve seres humanos, podem existir riscos mínimos ou indiretos, como a possibilidade da quebra de confidencialidade, desconforto e constrangimento. Desta forma, durante a entrevista com os participantes, se houver o risco de constrangimento por entender que as questões tenham foco mais pessoal e/ou não compreender a mesma, poderemos como medida atenuante, suspender a entrevista a qualquer momento. $\mathrm{O}$ participante poderá solicitar a qualquer momento esclarecimento sobre a pesquisa, e os dados serão tratados com mais absoluto sigilo.

Os benefícios da pesquisa será o maior esclarecimento acerca das práticas de cuidado na saúde 
mental, podendo propiciar um espaço de reflexão que oportuniza o conhecimento e elaboração de novas estratégias de cuidado. A pesquisa pode identificar fatores que sendo tratados possam contribuir para $o$ aprimoramento do serviço.

A pesquisa foi submetida ao Comitê de Ética e Pesquisa com Seres Humanos da UFF, obtendo aprovação através do Parecer de número 3.527.292; a Secretaria de Saúde do município em questão, aprovou a participação da equipe multidisciplinar.

\section{Resultados esperados}

Implantar a Educação Permanente em Saúde como ferramenta estratégica para reflexão crítica do processo de trabalho e desenvolvimento dos trabalhadores de saúde mental do município de pequeno porte do Estado do Rio de Janeiro.

\section{Referências bibliográficas}

1. Brasil. Lei $n^{0} 10.216$, de 6 de abril de 2001. Dispõe sobre a proteção e os direitos das pessoas portadoras de transtornos mentais e redireciona o modelo assistencial em saúde mental. Diário Oficial da União. 6 de abr. 2001.

2. Brasil. Portaria n ${ }^{\circ} 3.088$, de 23 de dezembro de 2011. Institui a Rede de Atenção Psicossocial para pessoas com sofrimento ou transtorno mental e com necessidades decorrentes do uso de crack, álcool e outras drogas, no âmbito do Sistema Único de Saúde (SUS). Diário Oficial da União. 2011.

3. Ministério da Saúde (BR). Portaria GM n³36, de 19 de fevereiro de 2002. Define e estabelece diretrizes para o funcionamento dos Centros de Atenção Psicossocial. Diário Oficial da União. 2002.

4. Amarante P. Saúde mental e atenção psicossocial.Rio de Janeiro, Editora Fiocruz, 2007.

5. Ceccim R,MacruzL. EPS emMovimento. Educação e trabalho em saúde: a importância do saber da experiência. 2014. [acesso em: 25 de novembro de 2015]. Disponível em: http://eps.otics.org/material/entrada-textos/educacaoe-trabalho-em-saude-a-importancia-do-saber-da-experiencia.

6. Fortuna CM, Silva SSd, Mesquita LPd, Matumoto S, Oliveira PS, Santana FR. A socioclínica institucional como referencial teórico e metodológico para a pesquisa em enfermagem e saúde. Texto \& contexto enferm. 2017;26(4). [acesso em: 25 de novembro de 2015]. Disponível em http:// dx.doi.org/10.1590/0104-07072017002950017. 